ZECCA, Turri Valignani = V. ZECCA, Turri Valignani. Ricerca delle sue antichità e note storiche artistiche sulla chiesa medioevale de' SS. Giovanni Evangelista e Vincenzo Martire, L'Aquila 1905.

Żelazowski, Honos bigae $=\mathrm{J}$. Żelazowski, Honos bigae. Le statue onorarie romane su biga (Światowit. Supplement series A. 7), Varsavia 2001.

ZeNodocchio, Viabilità = S. Zenodocchio, Antica viabilità in Abruzzo, L'Aquila 2008.
ZIMMER, Berufsdarstellungen = G. ZIMMER, Römische Berufsdarstellungen (Archäologische Forschungen 12), Berlin 1982.

Zinanni, Rocca di Botte = D. Zinanni, Da Rocca di Botte a Trevi. Pietro Eremita, l'uomo della speranza, Roma 1988. ZveTAIEFF, Inscriptiones Italiae Inferioris = I. ZveTAIEFF Inscriptiones Italiae Inferioris dialecticae, Mosquae 1886. ZVETAIEFF, Inscriptiones Italiae Mediae = I. ZVETAIEFF Inscriptiones Italiae Mediae dialecticae, Lipsiae 1884/85.

\title{
PERIODICA SERIESQVE ABBREVIATE LAVDATAE
}

In hoc catalogo periodica et series et editiones titulorum non finitae reperiri possunt

$A A=$ Archäologischer Anzeiger. Beiblatt zum Jabrbuch des Deutschen Archäologischen Instituts

Abh.d. Akad.d. Wiss. Göttingen, Phil.-hist. Kl. = Abhandlungen der Gesellschaft [der Akademie] der Wissenschaften in Göttingen, Philologisch-historische Klasse

Abruzzo = Abruzzo. Rivista dell'Istituto di Studi Abruzzesi

Acme = Acme. Annali della Facoltà di Filosofia e Lettere dell'Università statale di Milano

Act. . Class. = Acta Classica. Journal of the Classical Association of South Africa

Acta Ant. Compl. = Acta Antiqua Complutensia

Acta Ant. Hung. = Acta Antiqua Academiae Scientiarum Hungaricae

Acta Arch. Lov. = Acta Archaeologica Lovaniensia

Acta Arch. Slov. = Acta Archaeologica. Ljubljana, Académie slovène

Acta Inst. Rom. Finl. = Acta Instituti Romani Finlandiae

Acta Inst. Rom. Sueciae = Acta Instituti Romani Regni Sueciae

Acta Musei Napocensis = Acta Musei Napocensis. PrehistoryAncient History - Archaeology

Acta Musei Porolissensis = Acta Musei Porolissensis. Anuarul Muzeului de Istorie si Artă di Zală

$A E=$ L'Année Épigraphique. Revue des publications épigraphiques relatives à l'antiquité romaine

Aegyptus = Aegyptus. Rivista italiana di egittologia e di papirologia

Aequa $=$ Aequa. Rivista di studi e ricerche sul territorio degli Equi

AEspA = Archivo Español de Arqueología

Aevum $=$ Aevum. Rassegna di Scienze storiche linguistiche e filologiche

AGEA = Agenzia per le Erogazioni in Agricoltura

$\mathrm{AIPhO}=$ Annuaire de l'Institut de Philologie et d'Histoire Orientales et Slaves

AJA $=$ American Journal of Archaeology

AJAH $=$ American Journal of Ancient History

$A J P h=$ American Journal of Philology

Album pittorico $=$ Album pittorico letterario abruzzese

Alm. Molise = Almanacco del Molise

Altri itinerari $=$ Altri itinerari. Arte, letteratura, natura, storia, tradizione

Anal. Rom. Inst. Dan. = Analecta Romana Instituti Danici

Anc. Soc. = Ancient Society

Ann. "Claudio Faina" = Annali della Fondazione per il Museo "Claudio Faina"

Ann. della Fac. di Lettere e Filosofia (Cagliari) = Annali della Facoltà di Lettere, Filosofia e Magistero dell'Università degli Studi di Cagliari

Ann. della Fac. di Lettere e Filosofia (Macerata) $=$ Annali della Facoltà di Lettere e Filosofia dell'Università degli Studi di Macerat
Ann. della Fac. di Lettere e Filosofia (Perugia) = Annali della Facoltà di Lettere e Filosofia dell'Università degli Studi di Perugia

Ann. Geoph. $=$ Annals of Geophysics

Ann. Inst. = Annali dell'Instituto di Corrispondenza Archeologica

Ann. Ist. It. Stud. Stor. = Annali dell'Istituto Italiano per gli Studi Storici

Ann. Ist. Orient. Napoli = Annali dell'Istituto universitario orientale di Napoli. Archeologia e Storia Antica

Ann. Ist. Orient. Napoli Filol. = Annali dell'Istituto universitario orientale di Napoli. Sezione Filologica

Ann. Ist . Orient . Napoli Ling. = Annali dell'Istituto universitario orientale di Napoli. Sezione Linguistica

Ann. Pisa = Annali della Scuola Normale Superiore di Pis

Ann. Semin. Bari = Annali del Seminario giuridico economico della Regia Università di Bari

Ann. Univ. Sofia. Fac. Lettres = Annuaire de l'Université de Sofia, Faculté des Lettres

Annales Acad. Scient. Fennicae $=$ Annales Academiae Scientia rum Fennicae

Annales Acad. Scient. Fennicae, Diss. Human. Litt. = Annales Academiae Scientiarum Fennicae, Dissertationes Humanarum Litterarum

Annuario = Annuario. Associazione storica del Sannio Alifano ANRW = Aufstieg und Niedergang der römischen Welt. Geschichte und Kultur Roms im Spiegel der neueren Forschung

Ant. Afr. = Antiquités Africaines. Histoire et archéologie de l'Afrique du Nord

Ant. Alt. = Antichità Altoadriatiche

Ant. tard. = Antiquité tardive

Antichthon = Antichthon. Journal of the Australian Society for Classical Studies

Antike Welt = Antike Welt. Zeitschrift für Archäologie und Kunstgeschichte

Antiqua = Antiqua. Pubblicazioni dell'Archeoclub

Antiquitas = Antiquitas. Abhandlungen zur alten Geschichte Anz. Österr. Akad. Wiss. = Anzeiger der Österreichischen Akademie der Wissenschaften

Aquil. nostra $=$ Aquileia nostra. Rivista dell'Associazione nazionale per Aquileia

$A \mho R=$ Atene e Roma

Arch. Class. = Archeologia Classica

Arch. Ephem. = Archaiologike Ephemeris

Arch. Epigr. Mitt. Österr. = Archäologisch-Epigraphische Mittheilungen aus Österreich-Ungarn

Arch. Ért. = Archaeologiai Értesitó

Arch. Esp. Arq. = Archivo Español de Arqueología Arch. Glott. Ital. = Archivio Glottologico Italiano Arch. Lex. Gram. = Archiv für lateinische Lexicographie und Grammatik mit Einschluß des älteren Mittelalters 
Arch. Med. $=$ Archeologia Medievale

Arch. [Reale] Soc. Rom. Stor. Patria = Archivio della $[$ Reale] Società Romana di Storia Patria

Arch. Relig. $=$ Archiv für Religionsgeschichte

Arch. Sott. = Archeologia Sotterranea

Arch. Stor. Eccl. Umbria = Archivio per la Storia Ecclesiastica dell'Umbria

Arch. Stor. March. = Archivio Storico Marchigiano

Arch. Stor. Mol. $=$ Archivio Storico Molisano

Arch. Stor. Prov. Nap. = Archivio Storico per le province napoletane

Arch. Stor. Prov. Parm. $=$ Archivio Storico per le province parmensi

Arch. Stor. Sannio Alifano = Archivio Storico del Sannio Alifano

Arch. Ven. $=$ Archivio Veneto. Deputazione di Storia Patria per le Venezie

Archeo $=$ Archeo. Attualità del Passato. Istituto Geografico de Agostini

Archeologia $=$ Archeologia . Rocznik Instytutu Archeologii $i$ Etnologii Polskiej Akademii Nauk

Archeomolise $=$ Archeomolise. Storia, archeologia e antropologia del Molise

Arctos $=$ Arctos. Acta Philologica Fennica

Athenaeum $=$ Athenaeum. Studi di Letteratura e Storia dell'Antichità

ATTA = Atlante tematico di topografia antica

Atti Accad. Pontan. = Atti Accademia Pontaniana

Atti Accad. Rov. Agiati = Atti dell'Accademia Roveretana degli Agiati

Atti CESDIR = Atti del Centro Studi e Documentazione sull'Italia Romana

Atti Conv. Magna Grecia $=$ Atti del Convegno di Studi sulla Magna Grecia

Atti Conv. Stud. Macer. = Atti del Convegno di Studi Maceratesi

Atti Ist. Veneto, Scienze, Lett., Arti = Atti dell'Istituto Veneto di Scienze, Lettere ed Arti, Classe di Scienze morali, Lettere ed Arti

Atti Mem. Acc. Patavina = Atti e Memorie dell'Accademia Patavina di Scienze, Lettere ed Arti

Atti Mem. Arcadia = Atti e Memorie dell'Arcadia

Atti Mem. Bologna = Atti e Memorie della Deputazione di Storia Patria per le Provincie di Romagna. Bologna

Atti Mem. Marche = Atti e Memorie della Deputazione di Storia Patria per le Marche

Atti Mem. Modena = Atti e Memorie Deputazione di Storia Patria per le Antiche Provincie Modenesi

Atti Mem. Soc. Tiburtina = Atti e Memorie della Società Tiburtina di Storia e d'Arte

Atti Mem. Treviso = Atti e Memorie dell'Ateneo di Treviso

Atti Napoli = Atti dell'Accademia di Scienze Morali e Politiche. Società Nazionale di Scienze, Lettere ed Arti in Napoli

Atti Reale Accad. Arch. Napoli = Atti della Reale Accademia di Archeologia, Lettere e Belle Arti di Napoli

Atti Soc. Stor. Sannio = Atti della Società Storica del Sannio

Atti Terra Lavoro = Atti della Reale Commissione Conservatrice dei Monumenti ed Oggetti di Antichità e Belle Arti della Provincia di Terra di Lavoro

Aufidus = Aufidus. Rivista di scienza e didattica della cultura classica

Aurea Parma $=$ Aurea Parma. Rivista di storia, letteratura ed arte

BABesch = 1, 1926 - 44, 1969: Bulletin van de Vereeniging tot Bevordering der Kennis van de Antieke Beschaving te 's Gravenhage; 45, 1970 - 82, 2007: Bulletin Antieke Beschaving. Babesch. Annual Papers on Mediterranean Archaeology; 83, 2008 sq.: BABESCH. Annual Papers on Mediterranean Archaeology
$B A R=$ British Archaeological Reports

$B A V=$ Biblioteca Apostolica Vaticana

Bayer. Akad.d. Wiss., Phil.-hist. Kl., Abh., N. F. = Bayerische Akademie der Wissenschaften, Philologisch-historische Klasse. Abhandlungen, Neue Folge

Bayer. Vorg.-Bl. $=$ Bayerische Vorgeschichtsblätter

$B C A R=$ Bullettino della Commissione Archeologica Comunale di Roma

$B C H=$ Bulletin de Correspondance Hellénique

$B D A S P=$ Bullettino della Deputazione Abruzzese di Storia Patria

BHAC = Bonner Historia-Augusta-Colloquium

Bibl. Arch. = Bibliotheca archaeologica

Bibl. de l'École Haut. Ét. Sc. Hist. = Bibliothèque de l'École des Hautes Études, Sciences Historiques et Philologiques

Bibl. de l'École Haut. Ét. Sc. Rel. = Bibliothèque de l'École des Hautes Études, Sciences Religieuses

Bibl. de l'Inst. Franç. Naples = Bibliothèque de l'Institut Français de Naples

Bibl. Dep. Umbria = Biblioteca della Deputazione di Storia Patria per l'Umbria

Bibl. des Écoles Fr. d'Athènes et de Rome = Bibliothèque des Écoles Françaises d'Athènes et de Rome

Bibl. Stud. Antichi = Biblioteca di Studi Antichi

Boll. Antinori = Bollettino della Società di Storia Patria Anton Ludovico Antinori negli Abruzzi

Boll. Arch. = Bollettino di Archeologia

Boll. Arte $=$ Bollettino d'Arte del Ministero della Pubblica Istruzione

Boll. Ass. Ital. Cart. $=$ Bollettino della Associazione Italiana di Cartografia

Boll. Dep. Umbria = Bollettino della Deputazione di Storia Patria per l'Umbria

Boll. Dioc. Norcia $=$ Bollettino Diocesano di Norcia

Boll. Filol. Class. $=$ Bollettino di Filologia Classica

Boll. Mon. Mus. Gall. Pontif. = Bollettino. Monumenti, Musei $e$ Gallerie Pontificie

Boll. Mus. Imp. Rom. = Bollettino del Museo dell'Impero Romano

Boll. Reg. Dep. Umbria = Bollettino della Regia Deputazione di Storia Patria dell'Umbria

Boll. Stor. Crem. $=$ Bollettino storico cremonese

Boll. Stor. Foligno = Bollettino storico della Città di Foligno

Boll. Stor. Sub. = Bollettino storico-bibliografico subalpino

Boll. Stud. Lat. = Bollettino di Studi Latini

Bonner Jahrb. = Bonner Jahrbücher des Rheinischen Landesmuseums in Bonn und des Vereins von Altertumsfreunden im Rheinlande

$B R G K=$ Bericht der Römisch-Germanischen Kommission des Deutschen Archäologischen Instituts Berlin

Brill Studies Epigraphy $=$ Brill Studies in Greek and Roman Epigraphy

BTCGI = G. NenCI - G. VAllet (Ed.), Bibliografia topografica della colonizzazione greca in Italia e nelle isole tirreniche

Bull. Acad. Belgique = Bulletin de la Classe des Lettres et des Sciences Morales et Politiques de l'Académie Royale de Belgique

Bull. Arch. Crist. = Bullettino di Archeologia Cristiana

Bull. Arch. Nap. = Bullettino Archeologico Napoletano

Bull. Inst. = Bullettino dell'Instituto di Corrispondenza Archeologica. Roma

Bull. Inst. Class. Stud. = Bullettin of the Institute of Classical Studies

Bull. Ist. Belge Rome = Bulletin de l'Istitut Historique Belge de Rome

Bull. Seism. Soc. Amer. = Bulletin of Seismological Society of America 
Bull. Trav. Hist. = Bulletin Archéologique du Comité des Travaux Historiques et Scientifiques

Caeculus $=$ Caeculus. Papers in Mediterranean Archaeology and Greek of Roman Studies

Cahiers du Centre G. Glotz = Cabiers du Centre G. Glotz Revue d'Histoire Ancienne publiée par le Centre de Recherches G. Glotz, Sorbonne

Cataloghi $=$ Cataloghi dei musei locali e delle collezioni del Lazio

CEDANT = Centro di studi e ricerche sui Diritti Antichi

CeSDIR = Atti del Centro Studi e Documentazione sull'Italia Romana

Chiron = Chiron. Mitteilungen der Kommission für Alte Geschichte und Epigraphik des Deutschen Archäologischen Instituts

CISAM = Centro italiano di studi sull'alto medioevo

Civ. Catt. = La Civiltà Cattolica

Class. Ant. = Classical Antiquity

Class. Med. = Classica et Mediaevalia. Revue danoise de philologie et d'histoire

Class. Philol. $=$ Classical Philology

Class. Quart. = Classical Quarterly

Class. Review = Classical Review

Clio $=$ Clio. Rivista trimestrale di studi storici

Coll. de l'École Fr. de Rome = Collection de l'École Française de Rome

Coll. Latomus $=$ Collection Latomus

Coll. Sodalizio = Colloqui del Sodalizio tra studiosi dell'arte

Columb. Stud . Class. $=$ Columbia Studies in the Classical Tradition

Conoscenze $=$ Conoscenze. Rivista annuale della Soprintendenza Archeologica per i Beni Ambientali Architettonici Artistici e Storici del Molise

Conoscenze n. s. = Conoscenze. Rivista semestrale della Direzione Regionale per Beni Culturali e Paesaggistici del Molise

Considerazioni $=$ Considerazioni di Storia ed Archeologia

Contr. Ist. Fil. Class. = Contributi dell'Istituto di Filologia Classica. Pubblicazioni dell'Università Cattolica del Sacro Cuore

Contr. Valle Peligna $=$ Contributi alla cultura della Valle Peligna Superequana

CRAI = Comptes Rendus de l'Académie des Inscriptions et Belles-Lettres

CRDAC = Centro Ricerche e Documentazione sull'Antichità Classica. Monografie

Cronachetta mensuale $=$ Cronachetta mensuale di scienze naturali e d'archeologia redatta dal Prof. MARIANo Armellini

Cronichetta mensuale $=$ Cronichetta mensuale delle più importanti moderne scoperte nelle scienze naturali e loro applicazioni alle arti ed industria già redatta dalla $b . m$. di PIETRO Armellini continuata dal Prof. Tito Armellini e notizie archeologiche raccolte dal suo figlio MARIANo ARMELLINI

CSIR = Corpus Signorum Imperii Romani

Cultura Escrita $=$ Cultura Escrita \& Sociedad

Daidalos = Daidalos. Studi e ricerche del Dipartimento di scienze del mondo antico. Viterbo

DASP = Deputazione Abruzzese di Storia Patria

Dep. Umbria = Deputazione di storia patria per l'Umbria

Dial. Arch. = Dialoghi di Archeologia. Rivista semestrale

Dial. Hist. Anc. = Dialogues d'Histoire Ancienne

Dict. Antiq. $=$ Dictionnaire des antiquités grecques et romaines Diss. Pann. $=$ Dissertationes Pannonicae

Diss. Pontif. Accad. Arch. = Dissertazioni della Pontificia Accademia Romana di Archeologia

Diz. epigr. = Dizionario epigrafico di antichità romane
Documenti = Documenti di Antichità Italiche e Romane Documenti Abruzzo Teramano = Documenti dell'Abruzzo Teramano

Documenti e studi = Documenti e studi. Collana del Dipartimento di Scienze dell'antichità dell'Università di Bari. Sezione storica

$E A A=$ Enciclopedia dell'arte antica, classica e orientale $E C=$ Enciclopedia Cattolica

Echo $=$ Echo. Université de Lausanne. Institut d'archéologie et d'histoire ancienne

$E E=$ Ephemeris Epigraphica

$E O=$ Enciclopedia Oraziana

Eos $=$ Commentarii Societatis Philologae Polonorum

Ephem. Dac. $=$ Ephemeris Dacoromana

Epigr. Studien = Epigraphische Studien

Epigraphica $=$ Epigraphica. Periodico Internazionale di Epigrafia

$E P R O=$ Études Préliminaires aux Religions Orientales dans l'Empire romain

Erga - Logoi $=$ Erga - Logoi. Rivista di storia, letteratura, diritto e cultura dell'antichità

$E R I A C=$ Équipe de Recherche Interdisciplinaire sur les Aires Culturelles

Étud. . Class. = Les Études Classiques

Études = Études de philologie, d'archéologie et d'histoire anciennes

Eugesta $=$ Eugesta. Journal on Gender Studies in Antiquit

Euphrosyne = Euphrosyne. Revista de filologia clássica

Eutopia $=$ Eutopia . Commentarii novi de antiquitatibus totius Europae

$E V=$ Enciclopedia Virgiliana

Fast. Arch. = Fasti Archeologici. Annual Bulletin of Classical Archaeology

Forsch. Berichte $=$ Forschungen und Berichte

Forsch. Sklaverei $=$ Forschungen zur antiken Sklaverei

GASLA = Giornale abruzzese di scienze, lettere e arti

Gerión = Gerión. Revista de Historia Antigua

Giorn. arcadico = Giornale arcadico di scienze, lettere e arti

Giorn. Ital. Filol. $=$ Giornale Italiano di Filologia

Giorn. Scavi Pompei = Giornale degli Scavi di Pompei

Glotta $=$ Glotta. Zeitschrift für griechische und lateinische Sprache

$\Gamma \rho \alpha ́ \mu \mu \alpha \tau \alpha=\Gamma \rho \alpha ́ \alpha \mu \alpha \tau \alpha$. Rivista di cultura umanistica

$H A B E S=$ Heidelberger Althistorische Beiträge und Epigraphische Studien

Habis = Habis. Revista de la Universidad de Sevilla

Harvard Studies in Class. Philol. $=$ Harvard Studies in Classical Philology

Helikon = Helikon. Rivista di tradizione e cultura classica

Hephaistos = Hephaistos. Kritische Zeitschrift zur Theorie und Praxis der Archäologie, Kunstwissenschaft und angrenzender Gebiete

Hermes $=$ Hermes. Zeitschrift für klassische Philologie Hesperìa = Hesperìa . Studi sulla grecità di Occidente

Hisp. Ant. = Hispania Antiqua . Revista de Historia Antigua

Hisp. Epigr. = Hispania Epigraphica

Hist. Soc. Res. = Historical Social Research

Historia = Historia. Zeitschrift für Alte Geschichte

Historiká = Historiká. Studi di storia greca e romana

"Opmos = "Opmos. Ricerche di storia antica

IASA = International Association of Sound and Audiovisual Archives

Ichnia = Ichnia . Collana del Dipartimento di Scienze Archeologiche e Storiche dell'Antichità

$I C I=$ Inscriptiones Christianae Italiae septimo saeculo antiquiores 
ICVR, N. S. = Inscriptiones Christianae urbis Romae septimo saeculo antiquiores, Nova Series [cf. supra inter opera breviate laudata $\mathrm{p}$. CLII].

$I G=$ Inscriptiones Graecae

Il bene comune = Il bene comune. Arte, cultura e civiltà per il terzo millennio

Illin. Studies = Illinois Studies in Language and Literature

Il Territorio = Il Territorio. Rivista quadrimestrale di cultura e studi sabini

Incidenza dell'antico = Incidenza dell'antico. Dialoghi di storia greca

Incontri culturali $=$ Deputazione Abruzzese di Storia Patria. Incontri culturali dei soci

Index = Index. Ouaderni camerti di studi romanistici. International Survey of Roman Law

Indog. Bibl. = Indogermanische Bibliothek

Indog. Forsch. = Indogermanische Forschungen. Zeitschrift für Indogermanistik und allgemeine Sprachwissenschaft

Inscr. It. = Inscriptiones Italiae

Inst. Hist. Belge de Rome = Institute Historique Belge de Rome Insulae Diomedeae = Insulae Diomedeae. Collana di ricerche storiche e archeologiche

Inv. luc $=$ Invigilata lucernis

Itala Tellus = Itala Tellus. Studi di storia dell'Italia preromana e romana

Iura $=$ Iura. Rivista internazionale di diritto romano e antico

Jahreshefte d. Österr. Arch. Inst. = Jahreshefte des Österreichischen Archäologischen Instituts.

JAT vide Riv. Top. Ant.

$J C P h=$ Jabrbuch für classische Philologie

$J D A I=$ Jahrbuch des Deutschen Archäologischen Instituts

$J E S=$ The Journal of Epigraphic Studies

$J P h=$ Jabrbuch für Philologie

IRA = Journal of Roman Archaeology

$J R G Z M=J a b r b u c h$ des Römisch-Germanischen Zentralmuseums Mainz

$J R S=$ The Journal of Roman Studies

$J R T=$ Journal of Roman Topography

$J S=$ Journal des Savants

Kleine Texte $=$ Kleine Texte für theologische und philologische Vorlesungen und Übungen

Klio $=$ Klio. Beiträge zur alten Geschichte

Koinonia = Koinonia. Rivista dell'Associazione di Studi Tardoantichi

Ktéma = Ktéma . Civilisations de l'Orient, de la Grèce et de Rome antiques

$L A D=$ Laboratorio di studi e ricerche sulle antiche province danubiane. Università degli Studi di Ferrara. Facoltà di Lettere e Filosofia. Dipartimento di scienze storiche

L'Africa Romana = L'Africa Romana. Atti dei Convegni di studio su l' "Africa romana"

L'Ant. Class. = L'Antiquité classique

Lanx = Lanx. Rivista elettronica della Scuola di Specializzazione in Archeologia dell'Università degli Studi di Milano

Lares $=$ Lares. Quadrimestrale di studi demoetnoantropologici

Latium = Latium. Rivista di studi storici

Latomus = Latomus. Revue d'études latines

Lavori e Studi = Lavori e Studi della Soprintendeza per i Beni Archeologici del Lazio

$L F=$ Listy Filologicke

$L T L=$ Lexicon totius Latinitatis

Lumen = Il foglio di Lumen. Pubblicazione quadrimestrale dell'Associazione Culturale "Lumen"

Madr. Mitt. = Madrider Mitteilungen

Maia = Maia. Rivista di letterature classiche

Mare internum $=$ Mare internum. Archeologia e culture del mediterraneo
Mat. Disc. Test. Class. = Materiali e discussioni per l'analisi dei testi classici

Maurolico = Il Maurolico. Giornale di storia, scienze, lettere e arti

Mavors = Mavors. Roman Army Researche

MBPAR = Münchener Beiträge zur Papyrusforschung und antiken Rechtsgeschichte

Meander $=$ Meander. Miesiẹcznik poświẹcony kulturze świata starożytnego

Med. Antico = Mediterraneo Antico. Economie. Società. Culture. Rivista internazionale di Storia antica

MEFR = Mélanges d'Archéologie et d'Histoire de l'École Française de Rome

MEFRA = Mélanges de l'École Française de Rome. Antiquité MEFRM = Mélanges de l'École Française de Rome. Moyen $\hat{A} g e$

Mém. Acad. Inscr. Bell. Lett. = Mémoires présentés par divers savants à l'Academie des Inscriptions et Belles-Lettres

Mem. Accad. Lincei = Atti della Accademia Nazionale dei Lincei. Classe di Scienze morali, storiche e filologiche. Memo-

Mem. Amer. Acad. Rome = Memoirs of the American Academy in Rome

Mem. Conn. Acad. Art. Scien.$=$ Memoires of the Connecticut Academy of Arts and Sciences

Mem. Ist. Veneto $=$ Memorie dell'Istituto Veneto

Mem. Pontif. Accad. Arch. = Memorie della Pontificia Accademia Romana di Archeologia

Mem. Reale Accad. Napoli = Memorie della Reale Accademia di Archeologia, Lettere e Belle Arti di Napoli

Mem. Soc. Istriana = Atti e Memorie della Società Istriana di Archeologia e Storia Patria

Mém. Soc. nat. Antiq. France $=$ Mémoires de la Societé nationale des antiquaires de France

Metis = Metis. Revue d'anthropologie du monde grec ancien Metodi e prospettive $=$ Metodi e prospettive. Studi di Linguistica, Filologia, Letteratura

Min. Epigr. Papyr. = Minima Epigraphica et Papyrologica

Misc. Bibl. Ap. Vat. = Miscellanea Bibliothecae Apostolicae Vaticanae

Misc. Greca e Romana = Miscellanea Greca e Roma. Studi pubblicati dall'Istituto Italiano per la Storia Antica

Misc. Hadr. Med. = Miscellanea Hadriatica et Mediterranea

Mitt. Hist. Ver. Pfalz = Mitteilungen des Historischen Vereins der Pfalz

Mnemosyne $=$ Mnemosyne. A journal of classical studies

Mon. Accad. Lincei = Monumenti antichi pubblicati per cura della Accademia Nazionale dei Lincei

Mon. Ant . Crist. $=$ Monumenti di Antichità Cristiana

Monuments et Mémoires = Monuments et Mémoires publiés par l'Académie des Inscriptions et Belles-Lettres avec le concours de la Fondation Dourlans

Munera $=$ Munera . Collana di Studi storici sulla Tarda Antichità

Mus. Helv. = Museum Helveticum. Schweizerische Zeitschrift für klassische Altertumswissenschaft

Museo genti Abruzzo = Museo delle genti d'Abruzzo

Nouvelle Clio = La Nouvelle Clio. Revue mensuelle de la découverte historique

Novitas $=$ Novitas. Università Sulmonese della Libera Età

NS = Notizie degli Scavi di Antichità

Nuova Riv. Stor. $=$ Nuova Rivista Storica

Nuovi Quad. Ist. Arch. Perugia = Nuovi Quaderni dell'Istituto di Archeologia dell'Università di Perugia

Nuovo Bull. Arch. Crist. $=$ Nuovo Bullettino di Archeologia Cristiana

Oebalus = Oebalus. Studi sulla Campania nell'Antichita 
Österr. Akad. Wiss. Phil.-Hist. Klasse = Österreichische Akademie der Wissenschaften, Philosophisch-Historische Klasse

Oikumene = Oikumene. Studia ad historiam antiquam classicam et orientalem spectantem

Oltenia = Oltenia . Studii şi comunicări. Arheologie şi Istorie Veche

Opuscula Epigr. = Opuscula Epigraphica

Opuscula Inst. Rom. Finl. = Opuscula Instituti Romani Finlandiae

Opuscula Rom. = Opuscula Romana

Orizzonti $=$ Orizzonti. Rassegna di archeologia

Ostraka = Ostraka. Rivista di antichita

Palamedes = Palamedes. A Journal of ancient history

Panorami $=$ Panorami. Riflessioni, discussioni e proposte sul diritto e l'amministrazione

Pap. Mon. Am. Acad. Rome = Papers and Monographs of the American Academy in Rome

$P A w B=$ Potsdamer altertumswissenschaftliche Beiträge

PBSR $=$ Papers of the British School at Rome

PCIA = Popoli e Civiltà dell'Italia Antica

Pegasus = Pegasus. Berliner Beiträge zum Nachleben der Antike

Philol. Monogr. Amer. = Philological Monographs of the American Philological Association

Philippika = Philippika. Marburger altertumskundliche Abhandlungen

Philologus = Philologus. Zeitschrift für antike Literatur und ihre Rezeption

Phoenix = Phoenix. The Journal of the Classical Association of Canada

Picus = Picus. Studi e ricerche sulle Marche nell'antichità

Planus = Planus. Quaderni di Studio dell'Istituto Teologico abruzzese-molisano

Polymnia = Polymnia . Studi di storia romana

$P P=L a$ parola del passato. Rivista di studi antichi

Pragmateiai $=$ Pragmateiai. Collana di studi e testi per la storio economica, sociale e amministrativa del mondo antico

Publications Louvain = Publications d'histoire de l'art $e$ d'archéologie de l'Université Catholique de Louvain

Puteoli $=$ Puteoli. Studi di storia antica

QFIAB = Quellen und Forschungen aus italienischen Archiven und Bibliotheken

OuadAEI = Ouaderni del Centro di Studio per l'Archeologia Etrusco-Italica

Quad. Aquilano = Ente Provinciale per il Turismo - L'Aquila . Quaderni storico-artistici dell'Aquilano

Quad. Arch. Abruzzo = Quaderni di Archeologia d'Abruzzo. Notiziario della Soprintendenza per i Beni Archeologici dell'Abruzzo

Quad. Arte = I Quaderni dell'Arte

Quad. . Coldragonesi $=$ Quaderni Coldragones

Quad. Conoscenza = I Quaderni della Conoscenza

Ouad. Friul. Arch. = Ouaderni Friulani di Archeologia

Quad. Ist. Arch. Chieti = Quaderni dell'Istituto di Archeologia $e$ Storia antica dell'Università di Chieti

Quad. Ist. Glott. Bologna = Quaderni dell'Istituto di Glottologia dell'Università degli Studi di Bologna

Quad. Ist. Top. Roma = Quaderni dell'Istituto di Topografia Antica dell'Università di Roma

Quad. Ling. Urbino = Quaderni dell'Istituto di Linguistica dell'Università di Urbino

Quad. Museo Sulmona = Quaderni del Museo Civico di Sulmona

Ouad. Storia $=$ Quaderni di Storia

Quad. Storia Ant. Epigr. = Quaderni di Storia Antica e di Epigrafia

Quad. Ticin. = Quaderni Ticinesi di numismatica e antichità classich
Quad. Urb. = Quaderni Urbinati di cultura classica

RAAM = Rassegna d'arte degli Abruzzi e del Molis

RAC = Reallexicon für Antike und Christentum. Sachwörterbuch zur Auseinandersetzung des Christentums mit der Antiken Welt

RASA = Rassegna abruzzese di storia e arte

$R A S L=$ La Rivista abruzzese di scienze e lettere

RASLA = La Rivista abruzzese di scienze, lettere e arti

Rationes rerum $=$ Rationes rerum . Rivista di filologia e storia

$R C C M=$ Rivista di cultura classica e medievale

$R E=$ Paulys Realencyclopädie der classischen Altertumswissenschaft

REA = Revue des Études Anciennes

REG $=$ Revue des Études Grecques

REL $=$ Revue des Etudes Latines

$R G V V=$ Religionsgeschichtliche Versuche und Vorarbeiten

Rend. Accad. Arch. Napoli = Rendiconti della Accademia di Archeologia, Lettere e Belle Arti di Napoli

Rend. . Accad. Lincei = Atti della Accademia Nazionale dei Lincei. Classe di Scienze morali, storiche e filologiche. Rendiconti

Rend. Ist. Lomb. = Rendiconti dell'Istituto Lombardo. Classe di Lettere e Scienze Morali e Storiche

Rend. Pontif. Accad. Arch. $=$ Rendiconti della Pontificia Accademia Romana di Archeologia

RER $=$ Revue des Études Roumenes

Rev. Arch. $=$ Revue Archéologique

Rev. Arch. Narb. = Revue Archéologique de Narbonnaise

Rev. Belge de Philol. et d'Hist. $=$ Revue Belge de Philologie et d'Histoire

Rev. Hist. de Droit Fr. et Étr. = Revue Historique de Droit Française et Étranger

Rev. Intern. de Droits de l'Ant. = Revue Internationale de Droits de l'Antiquité

Rev. Num. = Revue Numismatique

Rev. Philol. = Revue de Philologie, de Littérature et d'Histoire Anciennes

Revista Bistriei $=$ Revista Bistriei. Un anuar de Complexul Muzeal Bistriţa Năsăud

Rhein. Mus. = Rheinisches Museum für Philologie

Rhesis $=$ Rhesis. International Journal of Linguistics, Philology and Literature

RIGI = Rivista Indo-Greco-Italica di filologia, lingua, antichit

RINASA = Rivista dell'Istituto Nazionale d'Archeologia e Storia dell'Arte

Riv. abruzzese $=$ Rivista abruzzese. Rassegna trimestrale di cultura

Riv. Arch. = Rivista di Archeologia

Riv. Arch. Como = Rivista archeologica dell'antica provincia e diocesi di Como

Riv. Arch. Crist. $=$ Rivista di Archeologia Cristiana

Riv. Filol. = Rivista di Filologia e Istruzione Classica

Riv. Stor. Ant. = Rivista di Storia Antica

Riv. Stor. Antichità = Rivista Storica dell'Antichità

Riv. Stor. Benedettina $=$ Rivista Storica Benedettina

Riv. Stor. Ital. = Rivista Storica Italiana

Riv. Stud. Liguri = Rivista di Studi Ligur

Riv. Stud. Pomp. = Rivista di Studi Pompeian

Riv. Top. Ant. = Rivista di Topografia Antica (Journal of Ancient Topography

$R M=$ Mitteilungen des Deutschen Archäologischen Instituts. Römische Abteilung

Roc. Hum. = Roczniki Humanistyczne

Salternum $=$ Salternum . Semestrale di informazione storica, culturale e archeologica a cura del Gruppo Archeologico Salernitano

Samnium = Samnium. Rivista storica 
Sandalion = Sandalion. Quaderni di cultura classica, cristiana e medievale

Sc. Ant. = Scienze dell'antichità. Rivista del Dipartimento di Scienze Storiche Archeologiche e Antropologiche dell'Antichità «Sapienza» Università degli Studi di Roma

Scripta antiqua $=$ Scripta antiqua. . Collection des éditions Ausonius

Scriptorium $=$ Scriptorium. Revue internationale des Études relatives aux manuscrits

SEBarc = Sylloge Epigraphica Barcinonensis

SEG $=$ Supplementum Epigraphicum Graecum

Seia $=$ Seia . Quaderni di studi storico-archeologici dell'Antichitè

Serta antiqua = Serta antiqua e mediaevalia . Pubblicazione dell'Università degli Studi di Genova, Dipartimento di Scienze dell'Antichità e del Medioevo

Sic. Gymn. = Siculorum Gymnasium

Signifer $=$ Signifer. Monografías y Estudios de Antigüedad Griega e Romana

$S L=$ Storia e Letteratura

Slavery and Abolition = Slavery and Abolition. A Journal of Slave and Post-Slave Studies

Soc. Scient. . Fennica = Societas Scientiarum Fennica

Soc. Scient. Fennica, Comment. Human. Litt. = Societas Scientiarum Fennica, Commentationes Humanarum Litterarum

Spoletium = Spoletium . Rivista di arte storia cultura

Stud. Ant. = Studi di Antichità

Stud. Arch. = Studia Archaeologica

Stud. Benev. $=$ Studi Beneventan $i$

Stud. . Class. Orient. = Studi Classici e Orientali

Stud. Doc. Hist. Iuris = Studia et Documenta Historiae et Iuris

Stud. Etr. = Studi Etruschi

Stud. Ist. It. Stor. Ant. = Studi pubblicati dal [Reale] Istituto per la Storia Antica / Studi pubblicati dall'Istituto Italiano per la Storia Antica

Stud. It. Filol. Class. = Studi Italiani di Filologia Classica

Stud. Macer. = Studi Macerates

Stud. Magr. = Studi Magrebini

Stud. . Merid. $=$ Studi Meridionali

Stud. . Orient. Crist.$=$ Studi sull'Oriente Cristano

Stud. Phil. Valentina = Studia philologica Valentina

Stud. Picen. $=$ Studia Picena

Stud. Ric. Matera = Siris. Studi e ricerche della Scuola di specializzazione in archeologia di Mater

Stud. Rom. = Studi Roman

Stud. Romagn. = Studi Romagnol

Stud. Saggi Ling. = Studi e Saggi Linguistici
Studi Ant. Crist. = Studi di antichità cristiana pubblicati a cura del Pontificio Istituto di Archeologia Cristiana

Studi Miscellanei $=$ Studi Miscellanei. Seminario di Archeologia e Storia dell' Arte Grega e Romana dell'Università di Roma

Suppl. Comm. Ateneo di Brescia $=$ Supplemento ai Commentari dell' Ateneo di Brescia

Suppl. It. = Supplementa Italica. Nuova serie.

$T A P h A=$ Transactions of the American Philological Association

Taras $=$ Taras. Rivista di archeologia

Temporis signa $=$ Temporis signa. Archeologia della tarda antichità e del medioevo

Testi e manuali = Testi e manuali per l'insegnamento universitario del Latino

ThCRA = Thesaurus Cultus et Rituum Antiquorum

ThLL $=$ Thesaurus linguae Latinae

Tituli $=$ Tituli. Pubblicazioni di epigrafia e antichità greche e romane dell'Università di Roma - "La Sapienza" (postea "Sapienza" - Università di Roma)

Topoi = Topoi Orient-Occident

Tyche $=$ Tyche. Beiträge zur alten Geschichte, Papyrologie und Epigraphik

Urbana species = Urbana species. Vita e città nell'Italia $e$ nell'Impero romano

Veleia = Veleia. Revista de prehistoria, historia antigua, arqueología y filología clásicas

Vestigia $=$ Vestigia. Beiträge zur alten Geschichte

Vesuviana = Vesuviana. An International Journal of Archaeological and Historical Studies on Pompeii and Herculaneum

Vet. Christ. $=$ Vetera Christianorum

Vetera $=$ Vetera . Ricerche di storia, epigrafia e antichità

Vichiana = Vichiana . Rassegna internazionale di Studi filologici e storici

WS = Wiener Studien. Zeitschrift für klassische Philologie und Patristik

Xenia $=$ Xenia. Semestrale di Antichità

$Z C P=$ Zeitschrift für celtische Philologie

Zeitschr. d. Aach. Gesch. = Zeitschrift des Aachener Geschichtsvereins

Zeitschr. d. Savigny-Stiftung, Rom. Abt. = Zeitschrift der SavignyStiftung für Rechtsgeschichte. Romanistische Abteilung

Zetemata = Zetemata. Monographien zur Klassischen Altertumswissenschaft

$Z P E=$ Zeitschrift für Papyrologie und Epigraphik 